\title{
A new method for in-flight intensity calibration of high-resolution EUV and FUV spectrometers
}

\author{
U. Feldman ${ }^{1}$, E. Landi ${ }^{1}$, G. A. Doschek ${ }^{2}$, and J. T. Mariska ${ }^{2}$ \\ 1 Artep, Inc, 2922 Excelsior Springs Ct, Ellicott City, MD 21042, USA \\ e-mail: landi@poppeo.nrl.navy.mil \\ 2 E.O. Hulburt Center for Space Research, Naval Research Laboratory, Code 7670, Washington DC 20375-5320, USA
}

Received 3 May 2005 / Accepted 14 June 2005

\section{ABSTRACT}

We introduce a new technique for the measurement of the in-flight relative intensity calibration of high resolution spectrometers. This technique makes use of the free-free radiation in hot, dense active regions and flares, and combines it with spectral line intensities in an iterative procedure. After a few iterations, the relative intensity calibration and the temperature of the emitting plasma are determined. The application of this technique to the EIS instrument on board the Solar-B satellite (launch in 2006) is discussed.

Key words. instrumentation: spectrographs - techniques: spectroscopic - methods: observational - methods: data analysis

\section{Introduction}

Imaging spectrometers in the Far Ultraviolet (FUV) and Extreme Ultraviolet (EUV) wavelength ranges are among the most important tools for the measurement of the physical parameters of plasmas in the solar upper atmosphere. The diagnostic use of line intensities, profiles, wavelengths, and intensity ratios provides crucial information on the plasma density, temperature, dynamic state, thermal structure and element composition necessary to benchmark theoretical models.

The usefulness of spectral observations for solar studies depends on accurate wavelength and intensity calibrations of the instrument. This is usually achieved prior to launch by using laboratory calibration facilities that test the instrument response against known radiation sources. Stresses during launch, as well as degradation both of the optics and of the detectors during the mission, require that the calibration be monitored on a regular basis after the instrument has begun its operational life. Since satellite-borne instruments do not include on board calibration sources, however, it is necessary to use their own observations, or comparisons with other instruments, to monitor the calibration.

The use of coronal emission lines emitted by the same upper level as an intensity calibration technique is known since a long time (i.e. Griffin \& McWhirter 1962; Heroux 1964), and has been generalized to all lines of known relative intensity by Neupert \& Kastner (1983). This method was applied by Brosius et al. (1998) to the calibration of the 1995 flight of the Solar EUV Rocket Telescope and Spectrograph (SERTS) in the 170-225 $\AA$ spectral range. Brosius et al. (1998) considered intensity ratios of $\mathrm{Fe}$ lines emitted by the same ion to reduce their dependence on potentially uncertain factors such as ion and element abundances. A similar study was carried out by Landi et al. (1997, 1999) and Del Zanna et al. (2001) with $\mathrm{SOHO} / \mathrm{CDS}$ data, resulting in the first accurate and complete intensity calibration for that instrument. They also used the differential emission measure (DEM) distribution to provide further constraints on the intensity calibration as a function of wavelength. It is important to note that intensity calibration techniques using line intensity ratios provide only the relative, wavelength-dependent calibration of the instrument, and only with great difficulty can they determine the absolute calibration, as for example was done by Del Zanna et al. (2001).

Line ratio calibration techniques suffer from three fundamental disadvantages:

1. uncertainties in atomic physics parameters involved in line intensity calculations;

2. a limited knowledge of the physical properties and the temporal stability of the emitting plasma; and

3. a limited availability of a sufficient number of lines of the same ion at different wavelengths to cover large portions of the instruments' wavelength range.

These three factors may limit the accuracy of the in-flight intensity calibration.

In the present work, we propose a new technique to determine the in-flight intensity calibration of a high resolution spectrometer that makes use of the free-free radiation observed in hot, dense active regions and during flares. The simultaneous presence of this radiation at all wavelengths, the relatively well understood atomic physics that generates it, and its moderate sensitivity to the plasma physical parameters, make it a 
valuable tool for in-flight calibration studies, overcoming all three difficulties mentioned above. To further refine results obtained with the free-free continuum, we also use spectral lines, with an iterative procedure that provides, as a by-product, measurements of the temperature of the emitting plasma. We also describe the application of this technique to the calibration of the Solar-B/EIS instrument, to be launched in August 2006.

In Sect. 2, we briefly discuss the free-free radiation and introduce the calibration technique. In Sect. 3, the Solar-B/EIS instrument is briefly described and the application of the calibration technique to EIS spectra is outlined. A summary of the technique is given in Sect. 4.

\section{Calibration method}

\subsection{Free-free emission}

The emission ( $\mathrm{erg} \mathrm{cm} \mathrm{cm}^{-3} \mathrm{~s}^{-1} \AA^{-1}$ ) of free-free radiation can be expressed as (Rybicki \& Lightman 1979)

$P_{f f}=2.0 \times 10^{-19}\left(\Sigma_{\mathrm{i}} Z_{\mathrm{i}}^{2} N_{\mathrm{i}}\right) N_{\mathrm{e}} T^{-\frac{1}{2}} \mathrm{e}^{-\frac{h c}{\lambda k T}} g_{f f}(T) \lambda^{-2}$

where $N_{\mathrm{e}}$ and $N_{\mathrm{i}}$ are electron and ion number densities, respectively, $Z_{\mathrm{i}}$ is the effective charge of the ion $i, h$ and $k$ are the Planck and Boltzmann constants, $T$ is the electron temperature in $\mathrm{K}, \lambda$ is the wavelength in $\AA$, and $g_{f f}(T)$ is the velocity-averaged Gaunt factor. The values of $g_{f f}(T)$ are of the order of unity when $\frac{h c}{\lambda k T} \simeq 1$ and are in the range of $1-5$ for $10^{-4} \leq \frac{h c}{\lambda k T} \leq 1$ (Rybicki \& Lightman 1979; Karzas \& Latter 1961). The bulk of the free-free emission is due to interactions of free electrons with hydrogen and, to a lesser extent, helium, while free-free emission from heavier elements is negligible.

As the plasma temperature increases from a typical coronal temperature $\left(\simeq 10^{6} \mathrm{~K}\right)$ towards flare temperatures $\left(\simeq 10^{7} \mathrm{~K}\right)$, the free-free emissivities for a particular wavelength will change only slightly due to the decrease of the $T^{-1 / 2}$ factor and to the increase in the $g_{f f}(T)$ value. Thus, over this order of magnitude temperature increase, the free-free emission at coronal temperatures (i.e. $10^{6} \leq T \leq 10^{7} \mathrm{~K}$ ) at a given wavelength will decrease only by a factor of 1.25-1.5. An example of such behavior is shown in Fig. 1. In this figure, free-free emissivities are shown as a function of wavelength, at several temperatures between upper transition region $(\log T=5.5, T$ in $\mathrm{K})$ to extremely high temperatures $(\log T=7.5, T$ in $\mathrm{K})$. The wavelength dependence over $100 \AA$ is less than $40 \%$ in all cases except for the lowest temperature considered $(\log T=5.5, T$ in $\mathrm{K}$ ), and the temperature dependence over one order of magnitude in temperature $\left(10^{6}\right.$ to $\left.10^{7} \mathrm{~K}\right)$ is less than $25 \%$.

It is clear that the weak dependence on temperature of the free-free radiation and its insensitivity to electron density and element abundances enables its use for intensity calibration purposes when the emitting plasma's properties are not known a priori. Also, the mild dependence on wavelength allows the use of free-free emission to calibrate spectrometers observing large spectral intervals, since the free-free emission will be detected over the whole spectral range with fairly uniform signalto-noise ratios.

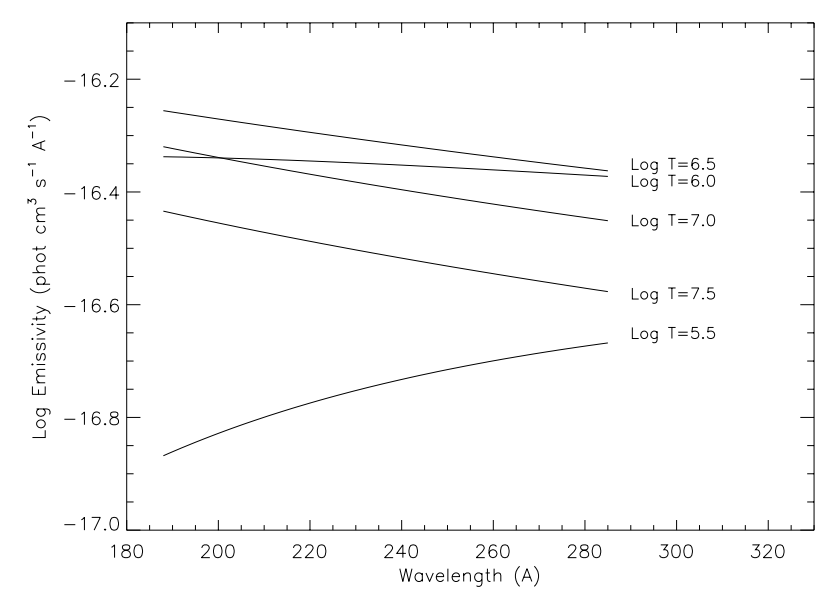

Fig. 1. Emissivity of the free-free continuum in the EUV spectral range, at several values of the $\log T$ of the plasma.

\subsection{The iterative calibration technique}

The free-free emission can be used for intensity calibration of high-resolution spectrometers in a four-step iterative process. It is essential that the resolution of the spectrometer be sufficiently high to provide spectral intervals where no lines are present, in order to avoid contamination of the free-free continuum with line emission.

As a preliminary step, the free-free count rates per unit time and wavelength are measured at many different wavelengths, in order to cover the entire spectral range of the instrument. Count rates of all lines are also measured from the spectrum. For a single temperature plasma, obtaining a calibration curve is straightforward.

- First step: the count rates $R$ of the free-free radiation at each wavelength are compared to predicted values $P$, calculated at a grid of temperatures encompassing a wide range of temperatures. The $R / P$ ratios are averaged over temperature; the standard deviation can be taken as a measure of the uncertainty of each $R / P$ ratio.

- Second step: all the $R / P$ ratios are normalized relative to one of them, resulting in a set of corrections to the observed count rates as a function of wavelength. An interpolation of such corrections with wavelength provides a first estimate of the relative calibration curve of the instrument. Note that the first time this procedure is carried out, it is not necessary to know the electron temperature of the emitting plasma.

- Third step: count rates of each spectral line are multiplied by the correction curve, resulting in relative-calibrated count rates. Ratios of count rates from lines of the same ion, or by consecutive stages of ionization of the same element, are used to measure the plasma temperature.

- Fourth step: once the temperature of the plasma is known, the predicted free-free emissivity $P$ is re-calculated using the measured temperature, and the new $P$ values are used in the first step to provide an improved correction curve.

The whole iterative procedure is thus re-applied until there is convergence of the calibration curve and of the measured temperature values. 
Table 1. Expected DN s ${ }^{-1}$ for several important lines in the two EIS channels. DN are calculated using the DEM of an M 2 flare, for a $1^{\prime \prime} \times 1^{\prime \prime}$ area on the Sun.

\begin{tabular}{rlclcr}
\hline \hline & Ion & Wvl. $(\AA)$ & Transition & $\log \left(T_{\mathrm{e}}\right)$ & $\mathrm{DN} \mathrm{s}^{-1}$ \\
\hline 1 & Fe X & 190.04 & $3 \mathrm{~s}^{2} 3 \mathrm{p}^{5}{ }^{2} \mathrm{P}_{1 / 2}-3 \mathrm{~s}^{2} 3 \mathrm{p}^{4}\left({ }^{1} \mathrm{D}\right) 3 \mathrm{~d}{ }^{2} \mathrm{~S}_{1 / 2}$ & 6.05 & 127 \\
2 & Fe XI & 188.22 & $3 \mathrm{~s}^{2} 3 \mathrm{p}^{4}{ }^{3} \mathrm{P}_{2}-3 \mathrm{~s}^{2} 3 \mathrm{p}^{3}\left({ }^{2} \mathrm{D}\right) 3 \mathrm{~d}{ }^{3} \mathrm{P}_{2}$ & 6.11 & 607 \\
3 & Fe XII & 195.12 & $3 \mathrm{~s}^{2} 3 \mathrm{p}^{3}{ }^{4} \mathrm{~S}_{3 / 2}-3 \mathrm{~s}^{2} 3 \mathrm{p}^{4}\left({ }^{3} \mathrm{P}\right) 3 \mathrm{~d}{ }^{4} \mathrm{P}_{5 / 2}$ & 6.15 & 3562 \\
4 & Fe XIII & 196.54 & $3 \mathrm{~s}^{2} 3 \mathrm{p}^{2}{ }^{1} \mathrm{D}_{2}-3 \mathrm{~s}^{2} 3 \mathrm{p} 3 \mathrm{~d}{ }^{1} \mathrm{~F}_{3}$ & 6.20 & 841 \\
5 & Fe XIV & 264.77 & $3 \mathrm{~s}^{2} 3 \mathrm{p}^{2} \mathrm{P}_{3 / 2}-3 \mathrm{~s}^{2} 3 \mathrm{p}^{2} \mathrm{P}_{3 / 2}$ & 6.27 & 1045 \\
6 & Fe XV & 284.15 & $3 \mathrm{~s}^{2}{ }^{1} \mathrm{~S}_{0}-3 \mathrm{~s} 3 \mathrm{p}^{1} \mathrm{P}_{1}$ & 6.33 & 5881 \\
7 & Fe XVI & 262.97 & $3 \mathrm{p}^{2} \mathrm{P}_{3 / 2}-3 \mathrm{~d}{ }^{2} \mathrm{D}_{5 / 2}$ & 6.40 & 2288 \\
8 & Ca XVII & 192.86 & $2 \mathrm{~s}^{2}{ }^{1} \mathrm{~S}_{0}-2 \mathrm{~s} 2 \mathrm{p}{ }^{1} \mathrm{P}_{1}$ & 6.70 & 12702 \\
9 & Fe XXIII & 263.77 & $2 \mathrm{~s}^{2}{ }^{1} \mathrm{~S}_{0}-2 \mathrm{~s} 2 \mathrm{p}{ }^{3} \mathrm{P}_{1}$ & 7.10 & 8151 \\
10 & Fe XXIV & 192.02 & $2 \mathrm{~s}^{2} \mathrm{~S}_{1 / 2}-2 \mathrm{p}{ }^{2} \mathrm{P}_{3 / 2}$ & 7.20 & 153714 \\
\hline
\end{tabular}

This iterative technique works if the plasma is isothermal but it can be used also where the plasma is multithermal. In this case, corrected count rates for the lines will be used in step three to determine the differential emission measure (DEM) of the emitting plasma, i.e. the distribution of plasma with temperature. In step four, the DEM will then be convolved with the free-free predicted emission as a function of $T$, to calculate improved values of $P$ and restart the iterative procedure.

This method can be applied any time the free-free radiation is visible in the spectrum. Usually the free-free continuum is observed during flares or in very hot and dense active regions, where the emission measure of the plasma is fairly high (i.e. $5 \times 10^{43}$ to $5 \times 10^{45} \mathrm{~cm}^{-3}$ - Landi et al. 2003; Feldman et al. 2003, 2004). Free-free emission in the EUV from bright active regions was recorded by the S082A spectroheliograph on Skylab (Tousey et al. 1977); examples of the emission are reproduced in the Atlas of Extreme Ultraviolet Spectroheliograms from 170 to $625 \AA$ (Feldman et al. 1987). Free-free emission has been detected in active regions and flares in the FUV by the SUMER spectrograph on board SOHO (Feldman et al. 2003), and is routinely observed in the X-rays during flares and in active regions.

\section{Application to EIS}

\subsection{The EIS instrument}

The EUV Imaging Spectrometer (EIS) is one of three instruments to be launched on the Japanese Solar-B Mission. A $0.5 \mathrm{~m}$ solar optical telescope (SOT) and an X-ray Telescope (XRT) are the two other instruments on the mission. The main goal of the Solar-B mission is to investigate the interaction between the Sun's magnetic field and its corona. Solar-B, which will be placed in a sun-synchronous orbit about the Earth, is scheduled to be launched in August 2006. The selected orbit will keep the instruments in nearly continuous sunlight, with no day/night cycling for nine months each year.

EIS is a normal incidence stigmatic spectrometer designed to simultaneously operate in the $170-210 \AA$ and $250-290 \AA$ wavelength bands. Radiation from the Sun enters the instrument through a thin aluminum filter that transmits the
EUV photons while stopping the visible light. The incoming photons are focused by a parabolic mirror on a slit. From there the radiation is dispersed by a toroidal concave grating and refocused on two back illuminated CCD detectors. A second thin Al filter is placed between the grating and the slit. To enhance the normal incidence reflectivity of EIS, two specially designed $\mathrm{Mo} / \mathrm{Si}$ multilayer coatings, one for the 170-210 $\AA$ channel the other for the 250-290 A channel were applied to the mirror and grating. The division between the two channels is achieved by dedicating half of the optical surfaces to one channel and half to the other. For the details of EIS design and observing plans see Culhane et al. (2005).

\subsection{Application of the diagnostic technique to EIS}

Reflectivity, transmission and quantum efficiency have been measured individually for the mirror, grating, filters and CCDs. Plots of the EIS effective area against wavelength for the two spectral ranges were produced (Seely et al. 2005). The resulting total instrumental efficiency has been used to predict the expected data numbers on the detector (DN) in lines emitted by an M 2 solar flare, using a typical DEM distribution $\varphi(T)$, from a $1^{\prime \prime} \times 1^{\prime \prime}$ area of the Sun in $1 \mathrm{~s}$ exposure time. The number of photons detected in each line is obtained by integrating over each line's contribution function $G(T)$ using the DEM curve, according to

$I=\frac{1}{4 \pi d^{2}} \int G(T) \varphi(T) \mathrm{d} T$

where $d$ is the Sun-Earth distance. To calculate line and continuum emissivities, we have used the CHIANTI atomic database (Dere et al. 1997; Young et al. 2003). Expected DN values for bright lines near the centers of the two EIS channels are listed in Table 1 for an M2 flare. Figure 2 is a plot of the emissivity as a function of temperature of the free-free together with the selected group of $\mathrm{Fe}$ and $\mathrm{Ca}$ lines listed in Table 1, which are expected to be present in the EIS range. The free-free emissivity is integrated over a $1 \AA$ band. The list includes one line from each of the Fe X-XVI, Ca XVII, Fe XXIII and Fe XXIV spectra. These ions sample coronal plasma both in active region and flare conditions, and allow temperature and DEM 


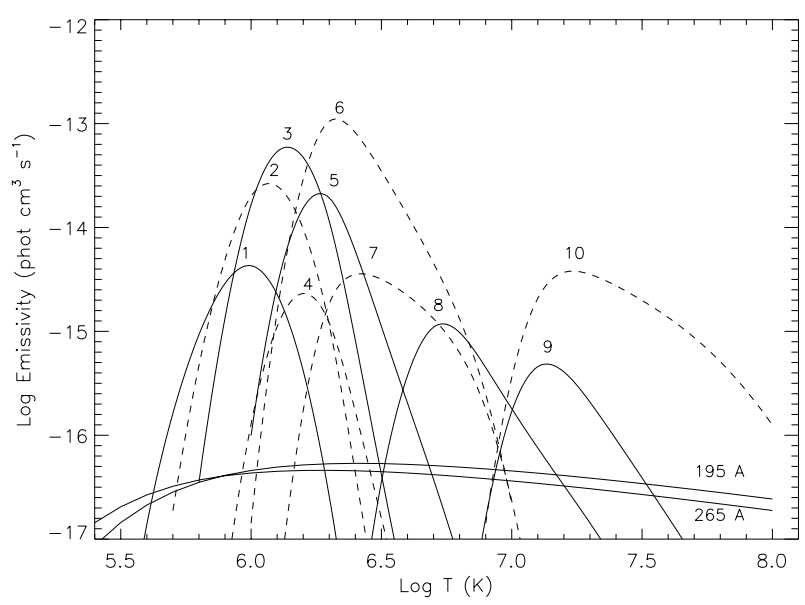

Fig. 2. Emissivity of the brightest lines in the EIS wavelength range and of the free-free continuum at two different wavelengths. Line numbers refer to the numbering of Table 1.

Table 2. Expected DN s ${ }^{-1} \AA^{-1}$ for the free-free and the He II recombination continuum calculated for an M2 flare in a $1^{\prime \prime} \times 1^{\prime \prime}$ area on the Sun.

\begin{tabular}{crr}
\hline \hline Wvl. $(\AA)$ & Free-free & Free-bound \\
\hline 175 & 3 & 0.1 \\
180 & 23 & 0.8 \\
185 & 114 & 4.3 \\
190 & 293 & 11.8 \\
195 & 444 & 18.9 \\
200 & 251 & 11.4 \\
205 & 46 & 2.2 \\
210 & 18 & 1.0 \\
\hline 250 & 35 & \\
255 & 56 & \\
260 & 78 & \\
265 & 102 & \\
270 & 117 & \\
275 & 100 & \\
280 & 64 & \\
285 & 37 & \\
290 & 21 & \\
\hline
\end{tabular}

diagnostics. Fe and $\mathrm{Ca}$ are low first ionization potential (FIP) elements ( $\mathrm{FIP} \leq 10 \mathrm{eV})$, so they are expected to follow the same pattern as all other low-FIP element abundance enhancement if any FIP effect (i.e. Feldman 1992) is present in the plasma. We can therefore eliminate any abundance variation in the solar corona in the determination of the relative calibration curve.

Table 2 lists the $\mathrm{DN} \mathrm{s}^{-1} \AA^{-1}$ for the free-free continuum at several wavelengths expected in the two EIS channels from an M 2 flare. Although the wavelength intervals where the freefree radiation can be measured will be more restricted than $1 \AA$, thus resulting in lower count rates (0.1-0.5 $\mathrm{A}$ at maximum), the order of magnitude of the count rates is high enough to be easily detected even with a $1 \mathrm{~s}$ exposure time. In the case of

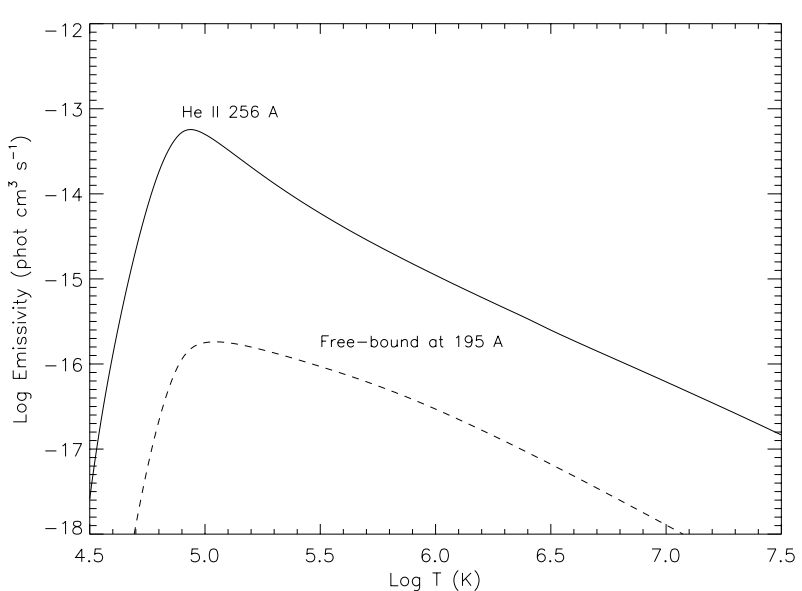

Fig. 3. Emissivity of the He free-bound continuum at $195 \AA$ and of the He II $256 \AA$ line as a function of temperature.

active regions the free-free emission is expected to be much lower so that the integration time needed to detect a sufficiently bright continuum will be longer, and also the emission a larger area will need to be summed. For example, count rates from a $10^{\prime \prime} \times 10^{\prime \prime}$ area will need an integration time of $10 \mathrm{~s}$ in order to reach values similar to those in Table 2 .

\subsection{The contamination from the He II free-bound continuum}

Although the application of the calibration technique to EIS is straightforward, consideration should be given to the possibility that the free-free continuum in the short wavelength channel could be contaminated by the He II free-bound continuum. The He II series limit is at $\simeq 228 \AA$, resulting in a possible He II free-bound contribution to the short wavelength EIS channel when cold plasma is included in the field of view. Figure 3 displays the emissivity of the He II Lyman- $\beta$ lines at $256 \AA$ line, expected to be visible in the long wavelength EIS channel, and the free-bound He II emissivity integrated over $1 \AA$ band near $195 \AA$ A. From a comparison between Figs. 3 and 2 it is apparent that if the plasma temperature along the line of sight is higher than $3 \mathrm{MK}\left(\log T_{\mathrm{e}} \geq 6.5\right)$, the free-bound contribution will be less than $10 \%$ of the total continuum emission and can be neglected. However, when the plasma along the line of sight is not isothermal and substantial colder plasma is present, the contribution to the total continuum may by be significant. Table 2 lists the expected intensities of the He II recombination continuum in an M 2 flare observed on the solar disk, along with the free-free values, and shows that the latter are always much higher than the former at any wavelength.

The amount of He II free-bound continuum can be evaluated using the intensity ratio of the $256 \AA \mathrm{He}$ II line intensity in the long wavelength channel to the free-bound continuum in the short-wavelength channel. This ratio is expected to be $\simeq 40: 1$, or even more, over the applicable temperature range (Fig. 2). By monitoring the $256 \AA$ intensity, the contribution of the free-bound to the free-free continuum could be continuously assessed and subtracted from the observed continuum at 
each iteration of the calibration procedure. The accuracy of this subtraction improves at each step.

If the observations are carried out outside the solar disk, and no prominence is included in the field of view, the He II recombination continuum will be negligible since no cold plasma will be in the field of view. In such cases the relative intensity between the $256 \AA$ line and the free-free continuum could be used to derive the coronal helium abundance, as done, for example, by Feldman et al. (2005) in the FUV range recorded by the SUMER instrument on SOHO. In that case, the $\mathrm{Ba}-\gamma$ lines of He II at $\simeq 1084 \AA$ were used.

\section{Conclusions}

In the present work, we present a new technique for the determination of the in-flight relative calibration of high resolution spectrometers that makes use of the free-free radiation emitted by dense active regions and flares.

The mild sensitivity of the free-free emission at coronal temperatures on wavelength and temperature makes it an ideal tool to evaluate the relative calibration of spectrometers covering wide spectral ranges, using spectra from coronal plasmas of initially unknown temperature.

We also describe the application of this technique to the Solar-B/EIS spectrometer, and discuss corrections due to the presence of the He II recombination continuum in the short wavelength channel of that instrument.

Acknowledgements. The work of Enrico Landi is supported by the NNH04AA12I, W10,232 and NNG04ED07P NASA grants.

\section{References}

Brosius, J. W., Davila, J. M., \& Thomas, R. J. 1998, ApJ, 497, L113

Culhane, J. L., Harra, L. K., Doschek, G. A., et al. 2005, Adv. Space Res., in preparation

Del Zanna, G., Bromage, B. J. I., Landi, E., \& Landini, M. 2001, A\&A, 379, 708

Dere, K. P., Landi, E., Mason, H. E., Monsignori Fossi, B. C., \& Young, P. R. 1997, A\&AS, 125, 149

Feldman, U. 1992, Phys. Scr., 46, 202

Feldman, U., Purcell, J. D., \& Dohne, B. C. 1987, An atlas of Ultraviolet Spectroheliograms from 170 to $625 \AA$, Ref $91-4100$ (Washington: DC Naval Research Laboratory)

Feldman, U., Landi, E., Doschek, G. A., Dammasch, I., \& Curdt, W. 2003, ApJ, 593, 1226

Feldman, U., Dammasch, I., Landi, E., \& Doschek, G. A. 2004, ApJ, 609,439

Feldman, U., Landi, E., \& Laming, J. M. 2005, ApJ, 619, 1142

Griffin, W. G., \& McWhirter, R. W. P. 1962, Proc. Conf. in Optical Instruments and Techniques, ed. K. J. Habell (London: Chapmasn and Hall), 14

Heroux, L. 1964, Proc. Phys. Soc., 83, 121

Karzas, W., \& Latter, R. 1961, ApJS, 6, 167

Landi, E., Landini, M., Pike, C. D., \& Mason, H. E. 1997, Sol. Phys., 175,553

Landi, E., Del Zanna, G., Landini, M., et al. 1999, A\&AS, 135, 171

Landi, E., Feldman, U., Innes, D. E., \& Curdt, W. 2003, ApJ, 582, 506

Neupert, W. M., \& Kastner, S. O. 1983, Sol. Phys., 128, 181

Rybicki, G. B., \& Lightman, A. P. 1979, Radiative Processes in Astrophysics (New York: John Wiley \& Sons)

Seely, J. F., et al. 2005, ApJ, in preparation

Tousey, R., Bartoe, J.-D. F., Bruekcner, G. E., \& Purcell, J. D. 1977, Appl. Optics, 16, 870

Young, P. R., Del Zanna, G., Landi, E., et al. 2003, ApJS, 144, 135 